\title{
An Improved Algorithm for Computing All the Best Swap Edges of a Tree Spanner*
}

\author{
Davide Bilò ${ }^{1}$, Feliciano Colella ${ }^{2}$, Luciano Gualà ${ }^{3}$, Stefano Leucci ${ }^{4}$, \\ and Guido Proietti ${ }^{5}$
}

1 Università di Sassari, Italy

davide.bilo@uniss.it

2 Gran Sasso Science Institute, L'Aquila, Italy

feliciano.colella@gssi.it

3 Università di Roma "Tor Vergata", Italy

guala@mat .uniroma2.it

4 ETH Zürich, Switzerland

stefano.leucci@inf.ethz.ch

5 Università degli Studi dell'Aquila, and Istituto di Analisi dei Sistemi ed Informatica, CNR, Roma, Italy

guido.proietti@univaq.it

\begin{abstract}
A tree $\sigma$-spanner of a positively real-weighted $n$-vertex and $m$-edge undirected graph $G$ is a spanning tree $T$ of $G$ which approximately preserves (i.e., up to a multiplicative stretch factor $\sigma$ ) distances in $G$. Tree spanners with provably good stretch factors find applications in communication networks, distributed systems, and network design. However, finding an optimal or even a good tree spanner is a very hard computational task. Thus, if one has to face a transient edge failure in $T$, the overall effort that has to be afforded to rebuild a new tree spanner (i.e., computational costs, set-up of new links, updating of the routing tables, etc.) can be rather prohibitive. To circumvent this drawback, an effective alternative is that of associating with each tree edge a best possible (in terms of resulting stretch) swap edge - a well-established approach in the literature for several other tree topologies. Correspondingly, the problem of computing all the best swap edges of a tree spanner is a challenging algorithmic problem, since solving it efficiently means to exploit the structure of shortest paths not only in $G$, but also in all the scenarios in which an edge of $T$ has failed. For this problem we provide a very efficient solution, running in $O\left(n^{2} \log ^{4} n\right)$ time, which drastically improves (almost by a quadratic factor in $n$ in dense graphs!) on the previous known best result.
\end{abstract}

1998 ACM Subject Classification G.2.2 Graph Theory, Graph algorithms, Trees

Keywords and phrases Transient edge failure, Swap algorithm, Tree spanner

Digital Object Identifier 10.4230/LIPIcs.ISAAC.2017.14

\section{Introduction}

The problem of computing all the best swap edges (ABSE) of a tree has a long and rich algorithmic tradition. Basically, let $G=(V(G), E(G), w)$ be an $n$-vertex and $m$-edge 2-edgeconnected undirected graph, with edge-weight function $w: E(G) \rightarrow \mathbb{R}^{+}$, and assume we are given a spanning tree $T$ of $G$, which was computed by addressing some criterion (i.e.,

* A full version of the paper is available at https://arxiv.org/abs/1710.01516.

cc) (i) Davide Bilò, Feliciano Colella, Luciano Gualà, Stefano Leucci, and Guido Proietti

c. licensed under Creative Commons License CC-BY

28th International Symposium on Algorithms and Computation (ISAAC 2017).

Editors: Yoshio Okamoto and Takeshi Tokuyama; Article No. 14; pp. 14:1-14:13

Leibniz International Proceedings in Informatics

LIPICS Schloss Dagstuhl - Leibniz-Zentrum für Informatik, Dagstuhl Publishing, Germany 
objective function) $\phi$. Then, the problem is that of computing a BSE for every edge $e \in E(T)$, namely an edge $f \in E(G) \backslash E(T)$ such that the swap tree $T_{e / f}$ obtained by swapping $e$ with $f$ in $T$ optimizes some objective function $\phi^{\prime}$ out of all possible swap trees. Quite reasonably, the function $\phi^{\prime}$ must be related (if not coinciding at all) with $\phi$.

The first immediate motivation for studying an ABSE problem comes from the edge fault-tolerance setting - a commonly accepted framework. Broadly speaking, the algorithmic question here is to design sparse subgraphs that guarantee a proper level of functionality even in the presence of an edge failure. In such a context, the rationale of an ABSE-based solution is the following: operations are normally performed on a (possibly optimal) spanning tree, and whenever an edge failure takes place, a corresponding BSE is plugged in. This way, the connectivity is reestablished in the most prompt and effective possible way (see also $[13,19]$ for some additional practical motivations).

Besides their practical relevance, ABSE problems have also an interesting theoretical motivation. Indeed, swapping can be reviewed as an exploration of the space of the perturbed (w.r.t. an edge swap) solutions to a given spanning tree optimization problem. Thus, the algorithmic challenge of solving efficiently an ABSE problem is related with the understanding of the structure of this space of perturbed solutions. And this is exactly why each ABSE problem has its own combinatorial richness, and thus requires a specific approach to be solved efficiently. Then, different ABSE problems have required the use of completely different approaches and methods in order to obtain efficient solutions. For instance, the most famous and studied ABSE problem comes when $T$ is a minimum spanning tree (MST) of $G$. In this case, a best swap is of course a swap edge minimizing the cost (i.e., sum of the edge weights) of the swap tree, i.e., a swap edge of minimum weight (and we know this produces a MST of the perturbed graph). This problem is also known as the MST sensitivity analysis problem, and can be solved in $O(m \log \alpha(m, n))$ time [18], where $\alpha$ denotes the inverse of the Ackermann function, by using an efficient data structure, namely the split-findmin [11]. This was improving on another efficient solution given by Tarjan [21], running in $O(m \alpha(m, n))$ time and making use of the transmuter, namely a compact way of representing the cycles of a graph. Other data structures which revealed their usefulness to solve efficiently ABSE problems include kinetic heaps [6], top trees [3], mergeable heaps [17], and many others.

In this paper, we focus on the ABSE problem on the elusive spanning tree structure, namely the tree spanner (ABSE-TS problem in the following). A tree spanner is built with the aim of preserving node-to-node distances in $G$. Indeed, the stretch factor $\sigma$ of a spanning tree $T$ of $G$ is defined as the maximum, over all the pairs $u, v \in V(G)$, of $d_{T}(u, v) / d_{G}(u, v)$, where $d_{T}$ and $d_{G}$ denote distances in $T$ and $G$, respectively. Correspondingly, an optimal tree spanner has minimum stretch out of all the spanning trees of $G$. Unfortunately, finding an optimal tree spanner is notoriously an APX-hard problem, with no known o(n)-approximation. Hence, once a given solution undergoes a transient edge failure, the recomputation from scratch of a new (near) optimal solution is computationally unfeasible. Thus, swapping in a tree spanner is even more attractive than in general, and indeed the ABSE-TS problem was studied in [9], where the authors devised two solutions for both the weighted and the unweighted case, running in $O\left(m^{2} \log n\right)$ and $O\left(n^{3}\right)$ time, respectively, and using $O(m)$ and $O\left(n^{2}\right)$ space, respectively. However, there the authors assume that a BSE is an edge minimizing the stretch of the swap tree w.r.t. distances in the original graph $G$, and not in the graph $G$ deprived of $e$, say $G-e$. This contrasts with the general assumption (and the intuition) that the quality of a swap tree should be evaluated in the surviving graph. Hence, in [3] the authors resorted to such a standard setting, and provided two efficient linear-space solutions for both the weighted and the unweighted case, running in $O\left(m^{2} \log \alpha(m, n)\right)$ and 
$O(m n \log n)$ time, respectively, and both using linear space. Notice that from a computational point of view, as shown in [3], the two settings are substantially equivalent, so our solutions can be used to improve the results given in [9] as well.

\subsection{Our result}

In this paper, we present a new algorithm that solves the ABSE-TS problem in $O\left(n^{2} \log ^{4} n\right)$ time and $O\left(n^{2}+m \log ^{2} n\right)$ space. Thus, our solution improves on the running time of both the algorithms provided in [3], for weighted and unweighted graphs, respectively, whenever $m=\Omega\left(n \log ^{3} n\right)$. Most remarkably, for dense weighted graphs, the improvement is almost quadratic in $n$.

To put into focus our result, it is worth noticing that, as observed in [9], the estimation of the stretch of the swap tree induced by a single swap edge $f$ for a given failing edge $e$, would in principle ask for the evaluation of the stretch of $O(m)$ relevant pairs of nodes in $G$, namely the endvertices of all the non-tree edges that may serve as swap edge for $e$ besides $f$. And in fact, a critical edge for $f$ is the one whose endvertices maximize such a stretch out of these non-tree edges, and two swap edges will be essentially compared on the basis of their stretch w.r.t. their critical edge. This is basically the reason why both previous approaches take $\Omega\left(m^{2}\right)$ time. Thus, to avoid such a bottleneck, we drastically reduce, on the one hand, the number of candidate best swap edges, and on the other hand, the number of potential critical edges that need to be checked. More precisely, for each of the $n-1$ considered edges in $T$, we succeed in reducing to $O(n \log n)$ the number of best swap edge candidates, and for each one of them we just need to check $O\left(\log ^{2} n\right)$ possible critical edges. The key ingredients to reach such a goal are the following:

- A centroid decomposition of $T$, which consists of a log-depth hierarchical decomposition of the vertices in $T$; a careful use of such a decomposition, combined with a set of preprocessing steps that associate various information with the tree nodes, allows us to reduce the number of candidate BSEs and of their corresponding candidate critical edges. As far as we know, this is the first time that such a decomposition is used to solve an ABSE problem, and we believe it will possibly be useful in other contexts as well.

- The second ingredient is given by the dynamic maintenance of the upper envelopes of a set of linear functions. Each of these functions is associated with a non-tree edge, and whenever the failure of a given tree edge is considered, it expresses the stretch such a non-tree edge induces w.r.t. a variable candidate BSE. This way, when we have to find a critical edge for a given candidate BSE $f$, we have to select the maximum out of all the functions once they are evaluated in $f$. In geometric terms, this translates into the maintenance of the upper envelope of a set of functions, with the additional complication that, for consistency reasons, this set of functions must be suitably partitioned into groups according to the underlying centroid decomposition, and moreover these groups are dynamic, since they depend on the currently considered tree edge.

\subsection{Related work}

The research on tree spanners is very active, also due to the strong relationship with the huge literature on spanners, where distances in $G$ are approximately preserved through a sparse spanning subgraph. As mentioned before, finding an optimal tree spanner is a quite hard problem. More precisely, on weighted graphs, if $G$ does not admit a tree 1-spanner (i.e., a spanning tree with $\sigma=1$, which can be established in polynomial time [8]), then the problem is not approximable within any constant factor better than 2 , unless $\mathrm{P}=\mathrm{NP}[15]$. In terms of 
approximability, no non-trivial upper bounds are known, except for the $O(n)$-approximation factor returned by a minimum spanning tree (MST) of $G$. If $G$ is unweighted, things go slightly better. More precisely, in this case the problem becomes $O(\log n)$-approximable, while unless $\mathrm{P}=\mathrm{NP}$, the problem is not approximable within an additive term of $o(n)[10]$. Moreover, the corresponding decision problem of establishing whether $G$ admits a tree spanner with stretch $\sigma$ is NP-complete for every fixed $\sigma \geq 4$ (for $\sigma=2$ it is polynomial-time solvable [8], while for $\sigma=3$ the problem is open). Finally, it is known that constant-stretch tree spanners can be found for several special classes of (unweighted) graphs, like strongly chordal, interval, and permutation graphs (see [7] and the references therein).

Concerning the problem of swapping in spanning trees, this has received a significant attention from the algorithmic community. There is indeed a line of papers that address ABSE problems starting from different types of spanning trees. Just to mention a few, besides the MST, we recall the minimum diameter spanning tree (MDST), the minimum routing-cost spanning tree (MRCST), and the single-source shortest-path tree (SPT). Concerning the MDST, a best swap is instead an edge minimizing the diameter of the swap tree [12, 16], and the best solution runs in $O(m \log \alpha(m, n))$ time [6]. Regarding the MRCST, a best swap is clearly an edge minimizing the all-to-all routing cost of the swap tree [22], and the fastest solution for solving this problem has a running time of $O\left(m 2^{O(\alpha(n, n))} \log ^{2} n\right)$ [5]. Concerning the SPT, the most prominent swap criteria are those aiming to minimize either the maximum or the average distance from the root, and the corresponding ABSE problems can be addressed in $O(m \log \alpha(m, n))$ time [6] and $O\left(m \alpha(n, n) \log ^{2} n\right)$ time [20], respectively. Recently, in [4], the authors proposed two new criteria for swapping in a SPT, which are in a sense related with this paper, namely the minimization of the maximum and the average stretch factor from the root, for which they proposed an efficient $O\left(m n+n^{2} \log n\right)$ and $O(m n \log \alpha(m, n))$ time solution, respectively.

Finally, for the sake of completeness, we mention that for the related concept of average tree $\sigma$-spanners, where the focus is on the average stretch w.r.t. all node-to-node distances, it was shown that every graph admits an average tree $O(1)$-spanner [1].

\subsection{Preliminary definitions}

Let $G=(V(G), E(G), w)$ be a 2-edge-connected, edge-weighted, and undirected graph with cost function $w: E(G) \rightarrow \mathbb{R}^{+}$. We denote by $n$ and $m$ the number of vertices and edges of $G$, respectively. If $X \subseteq V(G)$, let $E(X)$ be the set of edges incident to at least one vertex in $X$. When $X=\{v\}$, we may write $E(v)$ instead of $E(\{v\})$. Given an edge $e \in E(G)$, we will denote by $G-e$ the graph obtained from $G$ by removing edge $e$. Similarly, given a vertex $v \in V(G)$, we will denote by $G-v$ the graph obtained from $G$ by removing vertex $v$ and all its incident edges. Given an edge $e \in E(T)$, we let $S(e)$ be the set of all the swap edges for $e$, i.e., all edges in $E(G) \backslash\{e\}$ whose endpoints lie in two different connected components of $T-e$. We also define $S(e, X)=S(e) \cap E(X)$, and $S(e, X, Y)=S(e) \cap E(X) \cap E(Y)$. When $X=\{v\}$, we will simply write $S(e, v)$ in lieu of $S(e,\{v\})$. For any $e \in E(T)$ and $f \in S(e)$, let $T_{e / f}$ denote the swap tree obtained from $T$ by replacing $e$ with $f$.

Given two vertices $x, y \in V(G)$, we denote by $d_{G}(x, y)$ the distance between $x$ and $y$ in $G$. We define the stretch factor of the pair $(x, y)$ w.r.t. $G$ and $T$ as $\sigma_{G}(T, x, y)=\frac{d_{T}(x, y)}{d_{G}(x, y)}$. Accordingly, the stretch factor $\sigma_{G}(T)$ of $T$ w.r.t. $G$ is defined as $\sigma_{G}(T)=\max _{x, y \in V(G)} \sigma_{G}(T, x, y)$.

- Definition 1 (Best Swap Edge). An edge $f^{*} \in S(e)$ is a best swap edge (BSE) for $e$ if $f^{*} \in \arg \min _{f \in S(e)} \sigma_{G-e}\left(T_{e / f}\right)$.

In the sequel, in order to solve the ABSE-TS problem, we will show how to efficiently find a BSE for every edge $e$ of a tree spanner $T$ of $G$. 


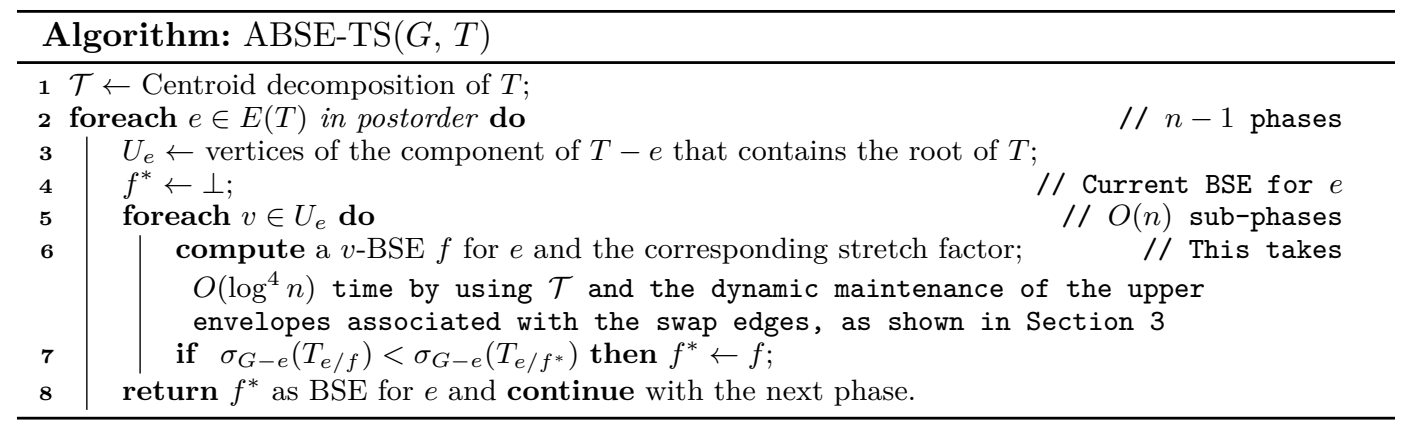

\section{High-level description of the algorithm}

It is useful to consider the tree $T$ as rooted at any fixed vertex, and to assume, w.l.o.g., that $T$ is binary. Indeed, if $T$ is not binary, then it is possible to transform $G$ and $T$ into an equivalent graph $G^{\prime}$ and a corresponding binary spanning tree $T^{\prime}$, with $\left|V\left(G^{\prime}\right)\right|=\Theta(n)$ and $\left|E\left(G^{\prime}\right)\right|=\Theta(m)$, and such that a BSE for any edge of $T$ is univocally associated with a BSE for a corresponding edge of $T^{\prime}$. This transformation uses standard techniques and can be performed in linear time.

As a preprocessing step, we compute a centroid decomposition of $T$. A centroid of an $n$-vertex tree is a vertex whose removal splits $T$ into subtrees of size at most $n / 2$ [14]. A centroid decomposition of $T$ can be computed in $O(n \log n)$ time, and can be represented by a tree $\mathcal{T}$ of height $O(\log n)$, whose nodes are actually subtrees of $T$. $\mathcal{T}$ is recursively defined as follows: the root of $\mathcal{T}$ is $T$. Then, let $\tau$ be a node of $\mathcal{T}$ (i.e., a subtree of $T$ ) such that $\tau$ contains more than one vertex, and let $c$ be a centroid of $\tau$. Since $T$ is binary, the forest $\tau-c$ contains at most 3 trees, that we call $\tau_{c}^{1}, \tau_{c}^{2}$, and $\tau_{c}^{3}$ (if $\tau-c$ generates less than 3 subtrees, we allow some $\tau_{c}^{i}$ to be the empty tree). Moreover, let $\tau_{c}^{0}$ be the subtree of $T$ containing the sole vertex $c$. Then, $\tau$ will have in $\mathcal{T}$ a child for each of the subtrees $\tau_{c}^{i}, i=0, \ldots, 3$ (see Figure $1(\mathrm{a}))$. Since a centroid on a $n$-vertex tree can be found in linear time, the whole procedure requires $O(n \log n)$ time, and it is easy to see that the height of $\mathcal{T}$ is $O(\log n)$.

Our solution (see Algorithm ABSE-TS) works in $n-1$ phases, one for each tree edge as considered in preorder w.r.t. $T$, and at the end of each phase returns a BSE for that edge. Let $e \in E(T)$ be the currently considered edge, and let $U_{e}$ (resp. $D_{e}$ ) be the set of vertices that belong to the connected component of $T-e$ that contains (resp. does not contain) the root of $T$. We break down each of these phases into $O(n)$ additional sub-phases: when edge $e$ is failing, we consider all the vertices in $U_{e}$ and, for each such vertex $v$, we solve a restricted version of the ABSE-TS problem where we compute: (i) a $v$-restricted best swap edge ( $v$-BSE for short), i.e., an edge $f \in \arg \min _{f \in S(e, v)} \sigma_{G-e}\left(T_{e / f}\right)$, and (ii) the corresponding stretch factor $\sigma_{G-e}\left(T_{e / f}\right)$. To simplify handling of special cases, whenever $S(e, v)=\emptyset$, we assume that $f=\perp$ and that $\sigma_{G-e}\left(T_{e / f}\right)=+\infty$. As we will see in the rest of the paper, the core of our algorithm is exactly the efficient computation of these $v$-BSEs and of their stretch factors. This is done trough a clever selection of a small set of candidate $v$-BSEs, as we will discuss in more detail in the next section. Once all the $v$-BSEs for $e$ are computed, a BSE for $e$ can be found as the one minimizing the associated stretch factor.

\section{Computing efficiently a $v$-BSE}

To show how a $v$-BSE for $e$ can be computed efficiently, we need some preliminary definitions: 


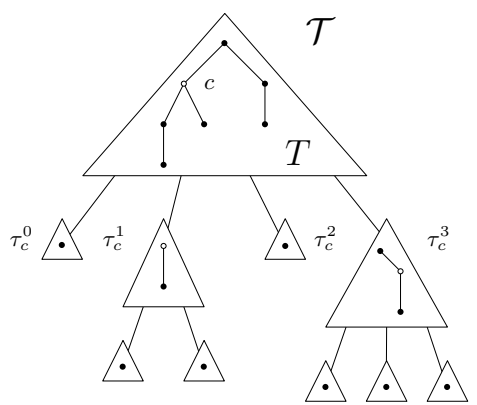

(a)

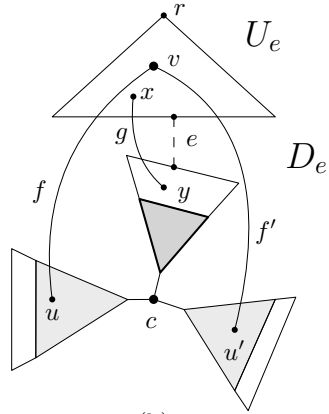

(b)

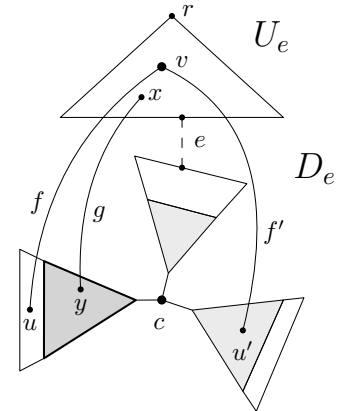

(c)

Figure 1 (a) An example of centroid decomposition of the tree $T$ (which corresponds to the first vertex of $\mathcal{T}$ ). (b) and (c): Two of the four possible cases situation illustrated in Lemma 5 . The subtree $\widehat{T}$ is represented by the three gray triangles along with the vertex $c$. $f$ is a swap edge for $e$ that minimizes $w(f)+d_{T}(u, c)$, and $g$ is its corresponding critical edge. The $(c, y)$-tree of $\widehat{T}$ is drawn in bold. Notice that $f$ and $g$ do not need to be incident to $\widehat{T}$.

- Definition 2 (Critical Edge). Given $e \in E(T)$ and a swap edge $f=(v, u) \in S(e, v)$, a critical $e d g e^{1}$ for $f$ is an edge $g=(x, y) \in S(e)$ maximizing $\phi(f, g):=\frac{d_{T}(x, v)+w(f)+d_{T}(u, y)}{w(g)}$.

- Definition 3 (Best Cut Edge). A v-best cut edge for $e$ ( $v$-BCE) is an edge $f \in S(e, v)$ minimizing $\varphi_{e}(f)=\max _{g \in S(e)} \phi(f, g)$.

Then, we will make use of the following property, which was given in [3]:

- Proposition 4. Every $v$-BCE for e is a $v$-BSE for $e$.

Let us first provide a high-level description of how we compute a $v$-BCE (i.e., a $v$-BSE) for $e$. The algorithm will compute $O(\log n) v$-BCE candidates, the best of which will be a $v$-BCE for $e$. Informally speaking, each candidate $f$ will be a swap edge close to the centroid of a certain subtree $\Lambda$ of $T$. Depending on the position of a critical edge for $f$, the algorithm will recurse on a subtree of $\Lambda$ and it will look for the next candidate. Thanks to the centroid decomposition of $T$, the number of recursions/candidates will then be $O(\log n)$.

The key ingredient for the correctness of our algorithm is the next lemma. Given a subtree $\widehat{T}$ of $T$, a vertex $c \in V(\widehat{T})$, and a vertex $y \in V(T)$, consider the first vertex $z$ of the unique path from $y$ to $c$ in $T$ that also belongs to $V(\widehat{T})$. The $(c, y)-$ tree of $\widehat{T}$ is defined as follows: (1) if $z=c$, then it is the empty tree; otherwise (2) it is the tree of the forest $\widehat{T}-c$ that contains $z$. Then, the following holds (see also Figure 1 (b) and (c)):

- Lemma 5. Let $\widehat{T}$ be a subtree of $T$ such that $V(\widehat{T}) \subseteq D_{e}$, and let $c \in V(\widehat{T})$. Moreover, let $f=(v, u) \in S(e, v)$ be a swap edge for e that minimizes $w(f)+d_{T}(u, c)$, and let $g=(x, y)$ be a critical edge for $f$. Assume that $S(e, v, V(\widehat{T}))$ contains a $v-B C E$ for $e$. If $f$ is not a $v$-BCE for e, then $S\left(e, v, V\left(T^{\prime}\right)\right)$ contains a $v$-BCE for $e$, where $T^{\prime}$ is the $(c, y)$-tree of $\widehat{T}$.

1 Notice that this definition does not contain $d_{G-e}(x, y)$ at the denominator, as expected, since it already incorporates the property stated in the forthcoming Proposition 4. 


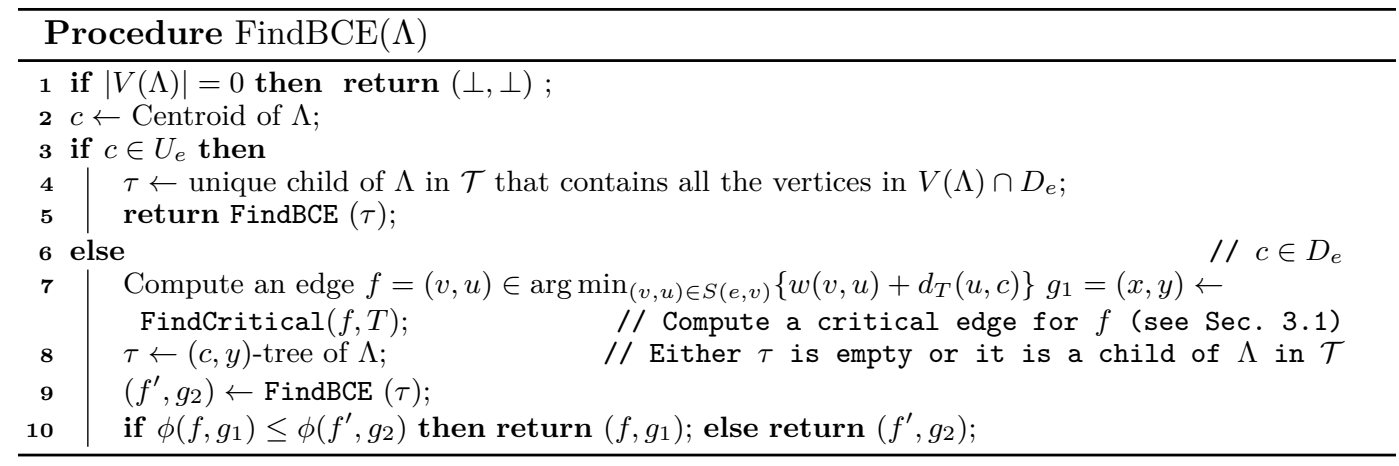

Proof. Suppose that $f$ is not a $v$-BCE for $e$, we will show that no swap edge $f^{\prime}=\left(v, u^{\prime}\right) \in$ $S(e, v)$ with $u^{\prime} \notin V\left(T^{\prime}\right)$ can be a $v$-BCE for $e$. Indeed:

$$
\begin{aligned}
\varphi\left(f^{\prime}\right) & \geq \phi\left(f^{\prime}, g\right)=\frac{d_{T}(x, v)+w\left(f^{\prime}\right)+d_{T}\left(u^{\prime}, y\right)}{w(g)} \\
& =\frac{d_{T}(x, v)+w\left(f^{\prime}\right)+d_{T}\left(u^{\prime}, c\right)+d_{T}(c, y)}{w(g)} \\
& \geq \frac{d_{T}(x, v)+w(f)+d_{T}(u, c)+d_{T}(c, y)}{w(g)} \geq \phi(f, g)=\varphi(f),
\end{aligned}
$$

where we used the fact that $d_{T}\left(u^{\prime}, y\right)=d_{T}\left(u^{\prime}, c\right)+d_{T}(c, y)$ as either $u^{\prime}=c$ or $u^{\prime}$ and $y$ are in two different connected components of $T-c$.

Lemma 5 allows us to design a recursive algorithm for computing a $v$-BCE for $e$, whose key steps are highlighted in Procedure FindBCE (notice that $v$ and $e$ are fixed). More precisely, the algorithm takes a tree $\Lambda$ of the centroid decomposition $\mathcal{T}$ such that $V(\Lambda) \cap D_{e} \neq \emptyset$, and it computes a pair $\left(f^{*}, g^{*}\right)$ such that if $S\left(e, v, V(\Lambda) \cap D_{e}\right)$ contains a $v$-BCE for $e$, then $f^{*}$ is a $v$-BCE for $e$, and $g^{*}$ is its critical edge. Procedure FindBCE makes use of an additional function FindCritical $(f, T)$ that returns a critical edge for $f$ w.r.t. the failure of $e$. The initial call will be $\operatorname{FindBCE}(T)$. In order to handle base cases, we assume $\phi(\perp, \perp)=+\infty$.

We now prove the correctness of the procedure:

- Lemma 6. Procedure FindBCE(T) computes a v-BCE for e.

Proof. Consider an invocation of the procedure and let $\Lambda$ and $\left(f^{*}, g^{*}\right)$ be its parameter and the edges it returns, respectively. We prove the following claim by induction on the cardinality of $V(\Lambda)$ : if $S\left(e, v, V(\Lambda) \cap D_{e}\right)$ contains a $v$-BCE for $e$, then $f^{*}$ is a $v$-BCE for $e$ and $g^{*}$ is a critical edge for $f^{*}$.

If $|V(\Lambda)|=0$, then the claim trivially holds. Otherwise, $|V(\Lambda)|>0$, and we distinguish two cases depending on the position of the centroid $c$ of $\Lambda$. If $c \in U_{e}$, then there is only one child $\tau_{c}^{j}$ of $\Lambda$ in $\mathcal{T}$ that contains all the vertices in $V(\Lambda) \cap D_{e}$, as otherwise the vertices in $D_{e}$ would be disconnected in $\Lambda$. Hence, if $S\left(e, v, V(\Lambda) \cap D_{e}\right)$ contains a $v$-BCE for $e$, then $S\left(e, v, V\left(\tau_{c}^{j}\right) \cap D_{e}\right)$ also contains a $v$-BCE for $e$, and the claim follows by the inductive hypothesis (as $\left|V\left(\tau_{c}^{j}\right)\right|<|V(\Lambda)|$ ). The remaining case is the one in which $c \in D_{e}$, here the claim follows from Lemma 5 (where now $\widehat{T}$ is the subtree of $T$ induced by $V(\Lambda) \cap D_{e}$ ) together with the inductive hypothesis.

Next lemma provides an upper bound to the running time of the procedure: 
- Lemma 7. Procedure FindBCE $(T)$ requires $O\left(\left(\Gamma_{f}+\Gamma_{\mathrm{FC}}\right) \log n\right)$ time, where $\Gamma_{f}$ and $\Gamma_{\mathrm{FC}}$ is the time required to perform Steps 7 and 7, i.e., the time to find edge $f$, and to execute Procedure FindCritical, respectively.

Proof. First of all, notice that Step 4 can be performed in $O(1)$ time, after a $O(\log n)$ preprocessing time in which we mark all the nodes of $\mathcal{T}$ on the path between the leaf of $\mathcal{T}$ containing the lower vertex of $e$ (which clearly belongs to $D_{e}$ ) and the root of $\mathcal{T}$. Then, we only need to bound the depth of the recursion of the call $\operatorname{FindBCE}(T)$. Observe that each time Procedure FindBCE $(\Lambda)$ recursively invokes itself on a tree $\Lambda^{\prime}$, we have that $\Lambda^{\prime}$ is a child of $\Lambda$ in $\mathcal{T}$. The claim follows since the height of $\mathcal{T}$ is $O(\log n)$.

Actually, the time to execute Step 7 is $O(\log n)$, after a preprocessing time and space of $O\left(n^{2}\right)$, by making use of top-trees [2]. Due to space limitations, the discussion of this result will appear in the full version of the paper. On the other hand, Procedure FindCritical will require $O\left(\log ^{3} n\right)$ time and $O\left(m \log ^{2} n\right)$ space, as we will show in the next two subsections.

\subsection{Computing a critical edge for $f$}

We will compute $O\left(\log ^{2} n\right)$ critical edge candidates for $f$ and we will show that a critical edge for $f$ will be one of them. More precisely, we look at $O(\log n)$ subtrees of the centroid decomposition $\mathcal{T}$ and, for each such subtree $\Lambda$, we will consider $O(\log n)$ subtrees $\Psi$ to find a critical edge candidate having one endpoint in $\Psi$ and the other in $\Lambda$. The choice of the $O\left(\log ^{2} n\right)$ pairs of trees is guided by the position of $f$, while the computation of a candidate for a given pair $(\Psi, \Lambda)$ is the core of the procedure and is described in the next subsection.

- Definition $8((\Psi, \Lambda)$-Critical Edge $)$. Given a failing edge $e$ and a swap edge $f=(v, u) \in$ $S(e, v)$, and given two trees $\Psi, \Lambda$ of the centroid decomposition $\mathcal{T}$, a $(\Psi, \Lambda)$-critical edge for $f$ is an edge $g=(x, y) \in \arg \max _{g^{\prime} \in S\left(e, V(\Psi) \cap U_{e}, V(\Lambda) \cap D_{e}\right)} \phi\left(f, g^{\prime}\right)$. When $\Psi=T$ we will refer to a $(\Psi, \Lambda)$-critical edge as a $\Lambda$-critical edge.

Let $f=(v, u) \in S(e, v)$ and let $\Lambda$ be a tree of the centroid decomposition $\mathcal{T}$ such that $u \in V(\Lambda)$. Procedure FindCritical returns a $\Lambda$-critical edge for $f$, when edge $e$ fails (such an edge always exists as $f$ has one endpoint in $U_{e}$ and the other in $\left.V(\Lambda) \cap D_{e}\right)$. Notice that the call FindCritical $(f, T)$ in Procedure FindBCE computes a critical edge for $f$, since a $T$-critical edge for $f$ is actually a critical edge for $f$.

Procedure FindCritical uses as a subroutine Procedure FindCriticalCandidate $(f, \Psi$, $\Lambda$ ), which for the sake of clarity will be described in the next subsection. For the moment, it suffices to know that FindCriticalCandidate receives three inputs, i.e., edge $f=(v, u)$ and two subtrees $\Psi, \Lambda$ of the centroid decomposition $\mathcal{T}$ such that $v \in \Psi$ and, either $u \notin V(\Lambda)$ or $\Lambda$ is the tree containing the sole vertex $u$, and it returns a $(\Psi, \Lambda)$-critical edge for $f$. If no such edge exists, then FindCriticalCandidate returns $\perp$ and we assume that $\phi(f, \perp)=-\infty$.

- Lemma 9. Let $f=(v, u) \in S(e, v)$, and let $\Lambda$ be a tree of the centroid decomposition $\mathcal{T}$ such that $u \in V(\Lambda)$. Procedure FindCritical $(f, \Lambda)$ returns a $\Lambda$-critical edge for $f$.

Proof. The proof is by induction on the cardinality of $V(\Lambda)$.

If $|V(\Lambda)|=1$, then the only vertex in $\Lambda$ must be $u$ and Procedure FindCritical invokes Procedure FindCriticalCandidate $(f, T, \Lambda)$. Hence, assuming such a procedure is correct, it returns a $(T, \Lambda)$-critical edge, i.e., a $\Lambda$-critical edge. If $|V(\Lambda)|>1$ then we distinguish two cases, depending on the position of the centroid $c$ of $\Lambda$.

If $c \in D_{e}$ it is sufficient to notice that a $\Lambda$-critical edge for $f$ must be incident to a tree $\tau_{c}^{i}$ for some $i=0,1,2,3$. Let $j$ be the unique index in $\{0,1,2,3\}$ such that $u \in V\left(\tau_{c}^{j}\right)$. If $j \neq i$ 


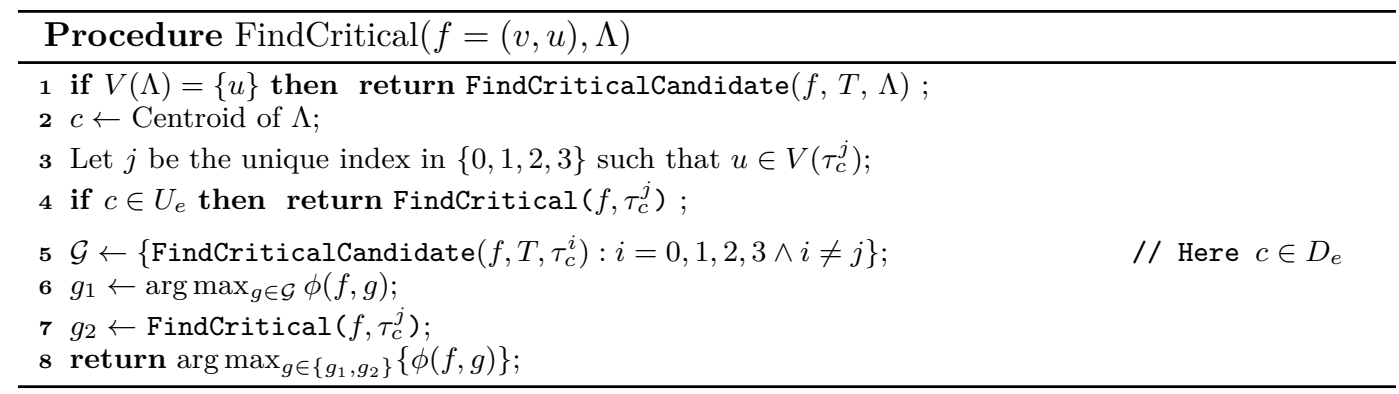

then, assuming Procedure FindCriticalCandidate is correct, it returns a $(T, \Lambda)$-critical edge $g_{1}$ (and hence a $\Lambda$-critical edge) for $f$. Procedure FindCritical then returns either $g_{1}$ or another edge $g$ such that $\phi(f, g)=\phi\left(f, g_{1}\right)$. If $j=i$, the algorithm is recursively invoked and, since $\left|V\left(\tau_{c}^{i}\right)\right|<|V(\Lambda)|$ we know, by the induction hypothesis, that it correctly returns a $\tau_{c}^{i}$-critical edge for $f$, which is also $\Lambda$-critical edge for $f$.

If $c \in U_{e}$, then we know that there is at most one $\tau_{c}^{i}$ containing one or more vertices in $D_{e}$ (as otherwise the vertices in $V(\Lambda) \cap D_{e}$ would be disconnected in $\Lambda$, a contradiction). Moreover, since $u \in V(\Lambda) \cap D_{e}$, there is exactly one such tree $\tau_{c}^{i}$, namely $\tau_{c}^{j}$. The algorithm recursively invokes itself on $\tau_{c}^{j}$ and, since $\left|V\left(\tau_{c}^{j}\right)\right|<|V(\Lambda)|$, we know, by induction hypothesis, that it returns a $\tau_{c}^{j}$-critical edge for $f$, which is also $\Lambda$-critical edge for $f$.

- Lemma 10. Procedure FindCritical $(f, \Lambda)$ requires $O\left(\Gamma_{\mathrm{FCC}} \cdot \log n\right)$ time, where $\Gamma_{\mathrm{FCC}}$ is the time required by an invocation of Procedure FindCriticalCandidate.

Proof. Notice that Procedure FindCritical performs exactly one recursive invocation for each vertex of the tree $\mathcal{T}$ on the unique path between the root of $\mathcal{T}$ and $u$ in $\mathcal{T}$. The claim follows since the height of $\mathcal{T}$ is $O(\log n)$.

In the next subsection, we show that $\Gamma_{\mathrm{FCC}}=O\left(\log ^{2} n\right)$, and then we give our final result.

\subsection{Procedure FindCriticalCandidate}

In this subsection, we describe the core of the procedure that computes a critical edge for $f$. Let us first describe informally the main idea of this part. Let $b \in U_{e}$ and $c \in D_{e}$, and consider any two edges $f=(v, u), g=(x, y) \in S(e)$ such that $b$ (resp. $c$ ) is on the unique path from $x$ to $v$ (resp. from $y$ to $u$ ) in $T$ (see Figure 2). It turns out that the stretch factor of any $f$ w.r.t. a given $g$ can be though as a linear function $\Phi_{b, c, g}(t)=\alpha_{b, c}(g) \cdot t+\beta_{b, c}(g)$, where $\alpha_{b, c}(g)$ and $\beta_{b, c}(g)$ only depend on $g$. More precisely, we will have that $\phi(f, g)=\Phi_{b, c, g}\left(t_{b, c}(f)\right)$, for a suitable value $t_{b, c}(f)$ which only depends on $f$. Hence, whenever we look for a critical edge for $f$, we can ask for a corresponding function $\Phi_{b, c, g}(t)$ with maximum value on $t_{b, c}(f)$. Since we do not know a priori the edge $f$ for which we need to compute a critical edge, we will maintain this information as the upper envelope of a suitable set of functions. Let us make this idea more precise.

Definition 11 (Upper Envelope). Let $\mathcal{F}=\left\{\Phi_{1}, \Phi_{2}, \ldots, \Phi_{\ell}\right\}$ be a finite set of functions, where $\Phi_{i}: \mathbb{R} \rightarrow \mathbb{R}$ for every $i=1,2, \ldots, \ell$. The upper envelope of $\mathcal{F}$ is defined as $\mathrm{UE}_{\mathcal{F}}: t \in$ $\mathbb{R} \mapsto \arg \max _{\Phi \in \mathcal{F}} \Phi(t) \in 2^{\mathcal{F}}$.

Let $b \in U_{e}$ and $c \in D_{e}$. Given an edge $f=(v, u)$, define $t_{b, c}(f)$ as the quantity $d_{T}(b, v)+w(f)+d_{T}(u, c)$. Given an edge $g=(x, y)$, define $\alpha_{b, c}(g)=\frac{1}{w(g)}$ and $\beta_{b, c}(g)=$ $\frac{d_{T}(x, b)+d_{T}(c, y)}{w(g)}$. Notice how, once $b$ and $c$ are fixed, $t_{b, c}(f)$ only depends on $f$ while $\alpha_{b, c}(g)$ and $\beta_{b, c}(g)$ only depend on $g$. Let $\Phi_{b, c, g}(t)=\alpha_{b, c}(g) \cdot t+\beta_{b, c}(g)$. 


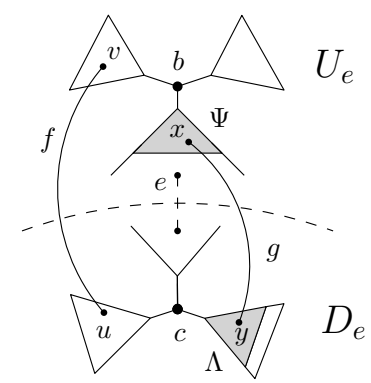

Figure 2 Illustration of Lemma 14. $f$ is a swap edge for $e, \Psi$ and $\Lambda$ are two trees of the centroid decomposition, and $b$ and $c$ are their corresponding parent centroids. $g$ is a potential $(\Psi, \Lambda)$-critical edge for $f$. Notice that the unique path from $x$ to $v$ (resp. from $y$ to $u$ ) passes through $b$ (resp. $c$ ).

- Lemma 12. Let $f=(v, u) \in S(e, v)$. Let $b \in U_{e}$ and $c \in D_{e}$. Let $X$ (resp. $Y$ ) be a set of vertices $x \in U_{e}$ (resp. $y \in D_{e}$ ) such that vertex $b$ (resp. $c$ ) is on the unique path from $x$ to $v$ (resp. from $y$ to $u$ ) in $T$. For every $g \in S(e, X, Y)$ we have $\phi(f, g)=\Phi_{b, c, g}\left(t_{b, c}(f)\right)$.

Proof. Let $g=(x, y)$. We have:

$$
\begin{aligned}
\phi(f, g) & =\frac{d_{T}(x, v)+w(f)+d_{T}(u, y)}{w(g)}=\frac{d_{T}(x, b)+d_{T}(b, v)+w(f)+d_{T}(u, c)+d_{T}(c, y)}{w(g)} \\
& =\frac{d_{T}(b, v)+w(f)+d_{T}(u, c)}{w(g)}+\frac{d_{T}(x, b)+d_{T}(c, y)}{w(g)}=\alpha_{b, c}(g) t_{b, c}(f)+\beta_{b, c}(g) \\
& =\Phi_{b, c, g}\left(t_{b, c}(f)\right) .
\end{aligned}
$$

- Definition 13 (Parent centroid). Let $\tau$ be a tree of the centroid decomposition $\mathcal{T}$. The parent centroid of $\tau$ is the centroid of the parent of $\tau$ in $\mathcal{T}$.

Lemma 12 is instrumental to proving the following (see Figure 2):

- Lemma 14. Let $f=(v, u) \in S(e, v)$, and let $\Psi, \Lambda$ be two trees of the centroid decomposition of $T$ such that the following conditions hold: (i) $v \notin V(\Psi)$ or $V(\Psi)=\{v\}$, and (ii) $u \notin V(\Lambda)$ or $V(\Lambda)=\{u\}$. Let b (resp. c) be the parent centroid of $\Psi$ (resp. $\Lambda$ ), and assume that $b \in U_{e}$ (resp. $c \in D_{e}$ ). Then, an edge $g$ is a $(\Psi, \Lambda)$-critical edge for $f$ if and only if $\Phi_{b, c, g} \in \mathrm{UE}_{\mathcal{F}}\left(t_{b, c}(f)\right)$ where $\mathcal{F}=\left\{\Phi_{b, c, g^{\prime}}: g^{\prime} \in S\left(e, V(\Psi) \cap U_{e}, V(\Lambda) \cap D_{e}\right)\right\}$.

Proof. First of all we show the following property of the centroid decomposition $\mathcal{T}$ : let $p, q \in V(T)$, and suppose that the unique path in $\mathcal{T}$ between the leaf nodes associated with $p$ and $q$ contains a node whose corresponding centroid is $z$. Then, the unique path between $p$ and $q$ in $T$ contains $z$. Indeed, if $z$ is either $p$ or $q$, the property is trivially true. On the other hand, suppose that $z \notin\{p, q\}$, and let $\tau$ be the subtree of $T$ associated with $z$ in $\mathcal{T}$. Then, let $\tau_{z}^{i}$ be the child subtree of $\tau$ containing $p$. Observe that $q$ is not in $\tau_{z}^{i}$. Moreover, by construction, each path from a node of $\tau_{z}^{i}$, and in particular from $p$, to any node outside $\tau_{z}^{i}$, and in particular to $q$, must pass through $z$.

We now prove the claim. If $V(\Psi)=\{v\}$ (resp. $V(\Lambda)=\{u\}$ ) then it follows from Lemma 12 by choosing $X=\{v\}$ and $Y=V(\Lambda) \cap D_{e}$ (resp. $X=V(\Psi) \cap U_{e}$ and $Y=\{u\}$ ). The complementary case is the one in which $v \notin V(\Psi)$ and $u \notin V(\Lambda)$. Consider the vertices $v$ and $b$ (resp. $u$ and $c$ ) in $\mathcal{T}$ and notice that $v$ (resp. $u$ ) cannot be an ancestor of $b$ (resp. $c$ ). Indeed, if that were the case, then the subtree of $T$ induced by the vertices in $V(\Psi)$ (resp. $V(\Lambda)$ ) would contain $b$ (resp. c) contradicting the hypothesis. Hence, the path from any vertex in $V(\Psi)$ to $v$ (resp. $V(\Lambda)$ to $u$ ) traverses $b$ (resp. $c$ ) in $\mathcal{T}$ and therefore the same holds in $T$. The claim follows by invoking Lemma 12 with $X=V(\Psi) \cap U_{e}$ and $Y=V(\Lambda) \cap D_{e}$. 


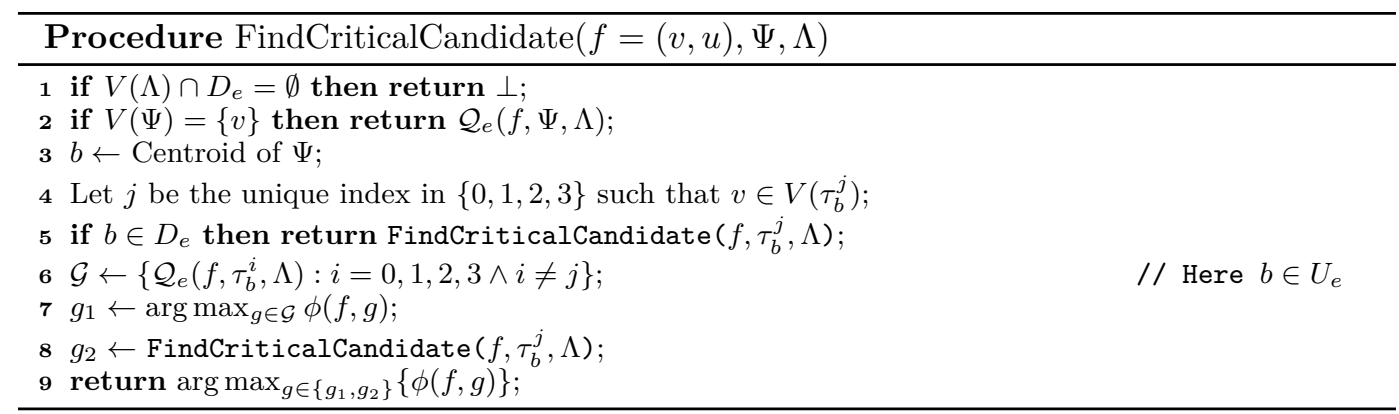

Lemma 14 allows us to design a recursive procedure to compute a $(\Psi, \Lambda)$-critical edge for $f$ (see Procedure FindCriticalCandidate). To this aim we will make use of a data structure $\mathcal{Q}_{e}$ that, for each edge $f \in S(e)$, and for each pair of trees $\Psi, \Lambda$ of the centroid decomposition, can perform a query operation that we name $\mathcal{Q}_{e}(f, \Psi, \Lambda)$. This query reports an edge whose function $\Phi_{b, c, g}$ is in $\mathrm{UE}_{\mathcal{F}}\left(t_{b, c}(f)\right)$ where $b$ and $c$ are the parent centroids of $\Psi$ and $\Lambda$, respectively, and $\mathcal{F}=\left\{\Phi_{b, c, g^{\prime}}: g^{\prime} \in S\left(e, V(\Psi) \cap U_{e}, V(\Lambda) \cap D_{e}\right)\right\}$.

Next two lemmas show the correctness and the running time of the procedure:

- Lemma 15. Let be given an edge $f=(v, u) \in S(e, v)$ and two trees $\Psi, \Lambda$ of the centroid decomposition such that: (i) $v \in V(\Psi)$, and (ii) $u \notin V(\Lambda)$ or $V(\Lambda)=\{u\}$. Then, Procedure FindCriticalCandidate $(f, \Psi, \Lambda)$ computes a $(\Psi, \Lambda)$-critical edge for $f$.

Proof. First of all notice that if $V(\Lambda) \cap D_{e}=\emptyset$, then the algorithm correctly returns $\perp$. We now prove the claim by induction on $|V(\Psi)|$. If $|V(\Psi)|=1$, then the only vertex in $\Psi$ must be $v$ and Procedure FindCriticalCandidate queries $\mathcal{Q}_{e}$ for $\mathcal{Q}_{e}(f, \Psi, \Lambda)$. By Lemma 14, the returned edge is a $(\Psi, \Lambda)$-critical edge for $f$. If $|V(\Psi)|>1$ then we distinguish two cases, depending on the position of the centroid $b$ of $\Psi$. If $b \in U_{e}$ it is sufficient to notice that a $(\Psi, \Lambda)$-critical edge for $f$ must be incident to a tree $\tau_{b}^{i}$ for some $i=0,1,2,3$. Let $j$ be the unique index in $\{0,1,2,3\}$ such that $v \in V\left(\tau_{b}^{j}\right)$. If $j \neq i$ then, by Lemma 14 , the query $\mathcal{Q}_{e}\left(f, \tau_{b}^{i}, \Lambda\right)$ returns a $\left(\tau_{b}^{i}, \Lambda\right)$-critical edge $g^{\prime}$ (and hence $g^{\prime}$ is also a $(\Psi, \Lambda)$-critical edge) for $f$. Procedure FindCritical then returns either $g^{\prime}$ or another edge $g$ such that $\phi(f, g)=\phi\left(f, g^{\prime}\right)$. If $j=i$, the algorithm is recursively invoked and, since $\left|V\left(\left|\tau_{b}^{i}\right|\right)\right|<|V(\Psi)|$ we know, by the induction hypothesis, that it returns a $\left(\tau_{b}^{i}, \Lambda\right)$-critical edge for $f$, which is also $(\Psi, \Lambda)$-critical edge for $f$. If $b \in D_{e}$, then there is at most one $\tau_{b}^{i}$ containing at least one vertex in $U_{e}$ (as the converse would imply that the vertices in $V(\Psi) \cap U_{e}$ are disconnected in $\Psi$, a contradiction). Moreover, since $v \in V(\Psi) \cap U_{e}$, there is exactly one such tree $\tau_{b}^{i}$, namely $\tau_{b}^{j}$. The algorithm recursively invokes itself on $\tau_{b}^{j}$ and we know, by induction hypothesis, that it returns a $\tau_{b}^{j}$-critical edge for $f$, which is also $(\Psi, \Lambda)$-critical edge for $f$.

- Lemma 16. Procedure FindCriticalCandidate $(f, \Psi, \Lambda)$ requires $O\left(\Gamma_{\mathcal{Q}_{e}} \cdot \log n\right)$ time, where $\Gamma_{\mathcal{Q}_{e}}$ is the time required by a query on $\mathcal{Q}_{e}$.

Proof. Notice that Procedure FindCriticalCandidate performs exactly one recursive invocation for each vertex of the tree $\mathcal{T}$ on the unique path between the root of $\mathcal{T}$ and $u$ in $\mathcal{T}$. The claim follows since the height of $\mathcal{T}$ is $O(\log n)$.

Thus, to get the promised running time of $O\left(\log ^{2} n\right)$ for $\Gamma_{\mathrm{FCC}}$, we are left to prove that $\Gamma_{\mathcal{Q}_{e}}=O(\log n)$. Actually, such a bound can be obtained by suitably implementing $\mathcal{Q}_{e}$ in such a way that all the underlying upper envelope functions are efficiently maintained. Due to 
space limitation, this technical part will appear in the full version of the paper. By combining all the lemmas, and by observing that we need $O\left(n^{2}\right)$ space to handle the top-trees, and $O\left(m \log ^{2} n\right)$ space to implement each $\mathcal{Q}_{e}$, we eventually can give the following:

- Theorem 17. The ABSE-TS problem can be solved in $O\left(n^{2} \log ^{4} n\right)$ time and $O\left(n^{2}+m \log ^{2} n\right)$ space.

\section{References}

1 Ittai Abraham, Yair Bartal, and Ofer Neiman. Embedding metrics into ultrametrics and graphs into spanning trees with constant average distortion. In Proc. of the 18th Annual ACM-SIAM Symposium on Discrete Algorithms, pages 502-511, 2007.

2 Stephen Alstrup, Jacob Holm, Kristian de Lichtenberg, and Mikkel Thorup. Maintaining information in fully dynamic trees with top trees. ACM Transactions on Algorithms, 1(2):243-264, 2005. doi:10.1145/1103963.1103966.

3 Davide Bilò, Feliciano Colella, Luciano Gualà, Stefano Leucci, and Guido Proietti. A faster computation of all the best swap edges of a tree spanner. In Proc. of the 22nd Intl. Colloquium Structural Information and Communication Complexity, pages 239-253, 2015. doi:10.1007/978-3-319-25258-2_17.

4 Davide Bilò, Feliciano Colella, Luciano Gualà, Stefano Leucci, and Guido Proietti. Effective edge-fault-tolerant single-source spanners via best (or good) swap edges. In Proc. of the 24th Intl. Colloquium Structural Information and Communication Complexity, in press.

5 Davide Bilò, Luciano Gualà, and Guido Proietti. Finding best swap edges minimizing the routing cost of a spanning tree. Algorithmica, 68(2):337-357, 2014. doi:10.1007/ s00453-012-9674-y.

6 Davide Bilò, Luciano Gualà, and Guido Proietti. A faster computation of all the best swap edges of a shortest paths tree. Algorithmica, 73(3):547-570, 2015. doi:10.1007/ s00453-014-9912-6.

7 Andreas Brandstädt, Victor Chepoi, and Feodor F. Dragan. Distance approximating trees for chordal and dually chordal graphs. J. Algorithms, 30(1):166-184, 1999. doi:10.1006/ jagm.1998.0962.

8 Leizhen Cai and Derek G. Corneil. Tree spanners. SIAM J. Discrete Math., 8(3):359-387, 1995. doi: 10.1137/S0895480192237403.

9 Shantanu Das, Beat Gfeller, and Peter Widmayer. Computing all best swaps for minimumstretch tree spanners. J. Graph Algorithms Appl., 14(2):287-306, 2010.

10 Yuval Emek and David Peleg. Approximating minimum max-stretch spanning trees on unweighted graphs. SIAM J. Comput., 38(5):1761-1781, 2008. doi:10.1137/060666202.

11 Harold N. Gabow. A scaling algorithm for weighted matching on general graphs. In Proc. of the 26th Annual Symposium on Foundations of Computer Science, pages 90-100, 1985. doi:10.1109/SFCS.1985.3.

12 Giuseppe F. Italiano and Rajiv Ramaswami. Maintaining spanning trees of small diameter. Algorithmica, 22(3):275-304, 1998. doi:10.1007/PL00009225.

13 Hiro Ito, Kazuo Iwama, Yasuo Okabe, and Takuya Yoshihiro. Polynomial-time computable backup tables for shortest-path routing. In Proc. of the 10th Intl. Colloquium Structural Information and Communication Complexity, pages 163-177, 2003.

14 Camille Jordan. Sur les assemblages de lignes. J. Reine Angew. Math, 70(185):81, 1869.

15 Christian Liebchen and Gregor Wünsch. The zoo of tree spanner problems. Discrete Applied Mathematics, 156(5):569-587, 2008. doi:10.1016/j.dam.2007.07.001.

16 Enrico Nardelli, Guido Proietti, and Peter Widmayer. A faster computation of the most vital edge of a shortest path. Inf. Process. Lett., 79(2):81-85, 2001. doi:10.1016/ S0020-0190(00)00175-7. 
17 Enrico Nardelli, Guido Proietti, and Peter Widmayer. Nearly linear time minimum spanning tree maintenance for transient node failures. Algorithmica, 40(2):119-132, 2004. doi : 10.1007/s00453-004-1099-9.

18 Seth Pettie. Sensitivity analysis of minimum spanning trees in sub-inverse-Ackermann time. In Proc. of the 16th Intl. Symposium on Algorithms and Computation, pages 964-973, 2005. doi:10.1007/11602613_96.

19 Guido Proietti. Dynamic maintenance versus swapping: An experimental study on shortest paths trees. In Proc. of the 4th Intl. Workshop on Algorithm Engineering, pages 207-217, 2000. doi:10.1007/3-540-44691-5_18.

20 Aleksej Di Salvo and Guido Proietti. Swapping a failing edge of a shortest paths tree by minimizing the average stretch factor. Theor. Comput. Sci., 383(1):23-33, 2007. doi: 10.1016/j.tcs.2007.03.046.

21 Robert Endre Tarjan. Sensitivity analysis of minimum spanning trees and shortest path trees. Inf. Process. Lett., 14(1):30-33, 1982. doi:10.1016/0020-0190(82)90137-5.

22 Bang Ye Wu, Chih-Yuan Hsiao, and Kun-Mao Chao. The swap edges of a multiple-sources routing tree. Algorithmica, 50(3):299-311, 2008. doi:10.1007/s00453-007-9080-z. 